
T. DUMONT ${ }^{1}$
S. LAZARE ${ }^{2}$
T. LIPPERT ${ }^{1, \infty}$
A. WOKAUN ${ }^{1}$

\title{
Changes in the etch rate of photosensitive polymers as a function of the pulse number
}

\author{
${ }^{1}$ Paul Scherrer Institut, 5232 Villigen, Switzerland \\ ${ }^{2}$ Laboratoire de Physico-Chimie Moléculaire, Université de Bordeaux1, 33405 Talence, France
}

\section{Received: 25 September 2003/Accepted: 24 February 2004 Published online: 26 July 2004 • ( ) Springer-Verlag 2004}

ABSTRACT The ablation rates of a polyimide and a triazene polymer were studied gravimetrically by a quartz micro balance for 248-nm and 308-nm irradiation. Special care was taken to examine the dependence of the ablation rate at constant fluences for single pulses and the influence of consecutive pulses at the same position. A clear trend was observed in these measurements, i.e., that the mass loss after the first pulse is always different from values for the following pulses. This implies that it is very difficult to determine true ablation rates, which are the foundation of most ablation models. The differences of the mass loss between the first pulse and the following pulses is most probably due to carbonization of the material, resulting in varying ablation rates for the following pulses. The ablation rates are thus not a real material property but a superposition of the material ablation rates with the ablation rates of carbon and carbonized material.

PACS 52.38.Mf; 71.20.Rv; 07.10.Lw

1

\section{Introduction}

UV laser ablation of polymers is the objective of intense experimental and theoretical research due to the potential applications in many fields (chemistry, physics, biology, and electronics) $[1,2]$. Studies of the laser-induced decomposition or transformation of polymers with single pulses and laser fluences close to the threshold of ablation require very sensitive techniques such as a quartz crystal microbalance (QCM) and atomic force microscopy (AFM). One important question in polymer ablation is related to the ablation mechanisms, i.e., photochemical versus photothermal. The understanding of these mechanisms is important for the design of new materials (e.g., photosensitive polymers) or the optimization of existing industrial processes. Models play an important role in the understannding of these mechanisms. The mass loss during laser ablation is one important parameter for the development of theoretical models that decribe ablation.

A mass loss (e.g., by QCM) due to photochemical reactions is expected for photosensitive polymers before a change

Fax: +41-563/104-412, E-mail: thomas.lippert@psi.ch in the surface morphology (e.g., by atomic force microscopy) can be measured. Another important parameter that may influence the ablation data is the role of solvent that remains in the polymer film (e.g., from the film preparation). Since QCM was first introduced as a gravimetric tool by Sauerbrey in 1959 [3], it has been used in many fields as sensors and thickness monitors but also as a gravimetric tool to study the ablation process of polymers [4]. The basis of the QCM is the fundamental vibration frequency of an AT-cut quartz plate that is directly proportional to its thickness. The frequency of the quartz decreases according to the amount of added mass by coating the surface with another material such as a polymer or a metal. The change can be directly converted to a mass load per unit area. If the density of the material is known, the film thickness of the material can be calculated. This method is very sensitive even to small variations of the mass load. For a 5-MHz quartz plate, a variation of $1 \mathrm{~Hz}$ corresponds to a mass variation of $17 \mathrm{ng} \mathrm{cm}^{-2}$. This corresponds for example to a thickness variation of $1.26 \AA$ of PET. Such high precision is especially useful for studying the ablation behavior of photosensitive polymers near the ablation threshold.

A polyimide (PMDA) and a triazene (TP) polymer (chemical structures shown in Scheme 1) were studied with QCM after irradiation with fluences below and above the threshold of ablation at irradiation wavelengths of 248 and $308 \mathrm{~nm}$. In detail, the dependency of the frequency change, and thus the ablation rate, on the number of pulses was examined. Küper et al. [4] performed similar experiments using a Kapton (or other polyimide). The measurements were performed in a vacuum chamber using just one coated quartz plate to perform the single-pulse and the consecutive-pulse experiments at the same position. However, carbonization of photosensitive polymers [5] and polyimide [6] has been reported and may very well influence the ablation behavior of the material. Since the chemical surface composition of polymers can be altered already in the first pulse, the ablation process for the consecutive pulses at the same spot can be different from the first pulse. The experimental data of Küper et al. are often used as experimental reference data for highly sophisticated models $[7,8]$. It is therefore of utmost importance to assure that the applied ablation rates are a true material property. 


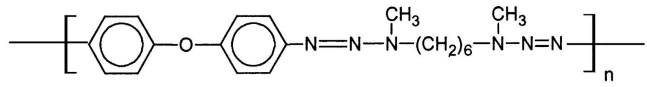

Triazene polymer (TP)

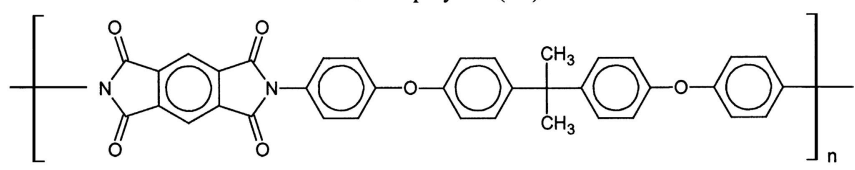

Polyimide polymer (PMDA)

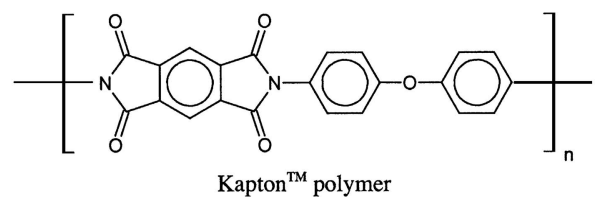

Scheme 1 Chemical structure of the applied polymers as well as of Kapton $^{\text {TM }}$

2

Experimental setup

Commercial quartz plates with silver electrodes and a fundamental frequency of $4.9152 \mathrm{MHz}$ were used in the QCM measurements. The samples were prepared by spin coating of several polymer layers on one side of the quartz plate. The polymer films were dried under nitrogen atmosphere at $40^{\circ} \mathrm{C}$ for at least $2 \mathrm{~h}$ for the triazene polymer and at $250^{\circ} \mathrm{C}$ for $1 \mathrm{~h}$ for the polyimide. The total film thickness of the polymers was in the range of several micrometers. The chemical structure of the polymers, as well as of Kapton, which is normally used in ablation studies, is shown in Scheme 1.

The aperture of the irradiation setup exceeded slightly the surface of the silver electrodes to assure that the complete sensitivity of the vibrating area was used. The laser fluence was varied by an attenuator and the beam energy was measured online via a beamsplitter and a powermeter. The frequency change over time was determined by a frequency counter connected to a computer.

The triazene polymer (TP) was ablated at irradiation wavelengths of 248 and $308 \mathrm{~nm}$. The polyimide (PMDA) was irradiated only at $308 \mathrm{~nm}$. Each sample was ablated at a constant fluence with one to ten pulses. The frequency change was measured after every pulse for at least $30 \mathrm{~s}$ at a rate of $1 \mathrm{~Hz}$. This allows one to determine whether the change in frequency, and thus the loss of mass of the polymer, remains constant after each pulse. All measurements were performed at ambient conditions.

\section{Results and discussion}

The changes of the frequency after each pulse were performed at different fluences. A clear deviation of the frequency change for consecutive pulses from the first pulse is visible for TP at 248-nm irradiation. For a constant removal of mass at a constant laser fluence, a constant frequency change for every laser pulse is expected. This is obviously not the case, as shown in Fig. 1. The frequency change for the following pulses is different from the first pulse. The curves in Fig. 1 show a clear trend of the frequency toward lower values, especially for higher fluences that cannot be explained by fluctuations of the laser energy. It can therefore be concluded that the ablation rates are clearly changing after the first pulse for high fluences. This means that less material is removed at the same fluence for pulse numbers $>1$ ( $>2$ for $124 \mathrm{mJcm}^{-2}$ ). A similar behavior was detected for TP at an irradiation wavelength of $308 \mathrm{~nm}$. The difference of the frequency change between the two irradiation wavelengths is only significant at fluences slightly above the threshold, between 27 and $43 \mathrm{mJcm}^{-2}$, and cannot be observed at higher fluences. The reason for this difference is that carbonization of TP is much more pronounced for irradiation at $248 \mathrm{~nm}$ [5].

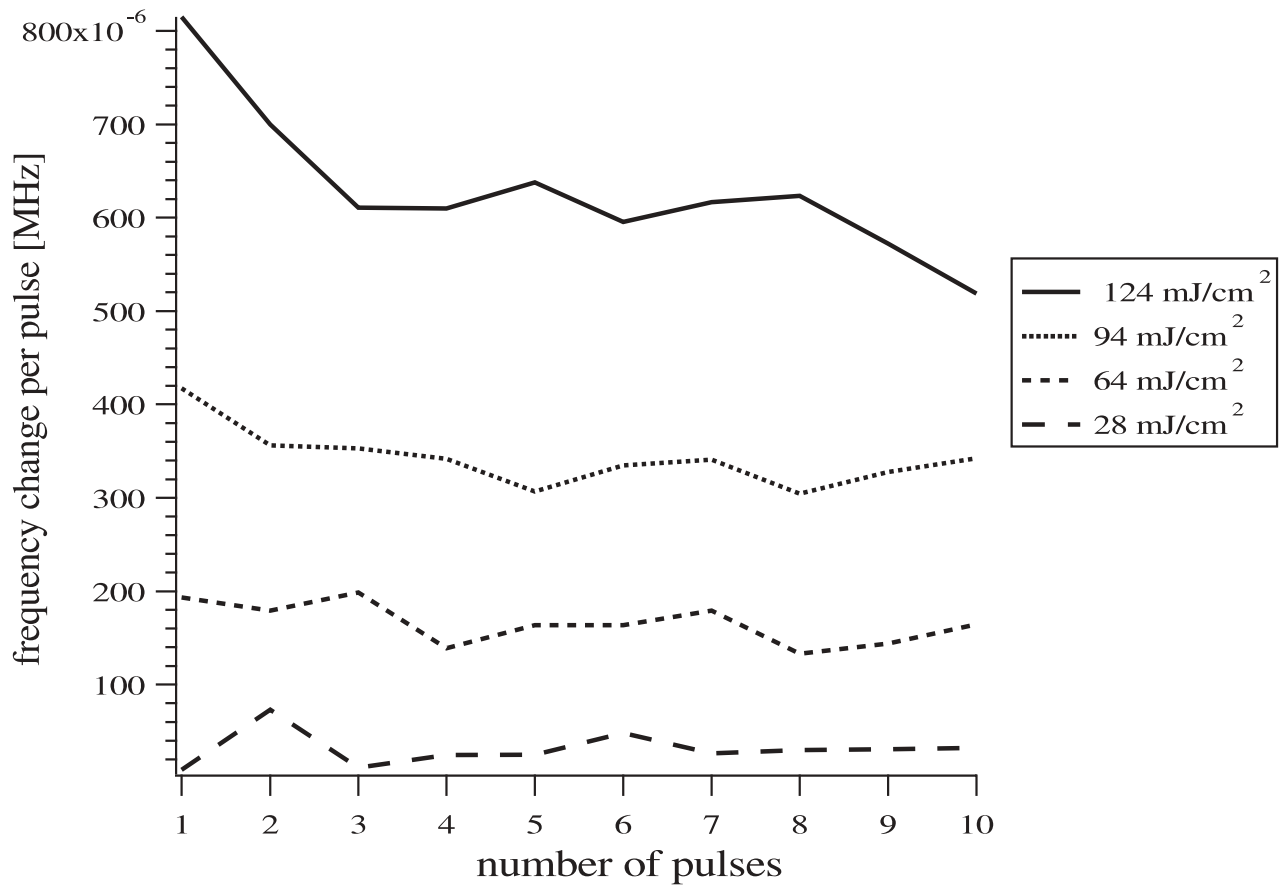

FIGURE 1 Frequency change between pulses at different fluences for TP at an irradiation wavelength of $248 \mathrm{~nm}$. One sample was used for each measurement at given fluence 

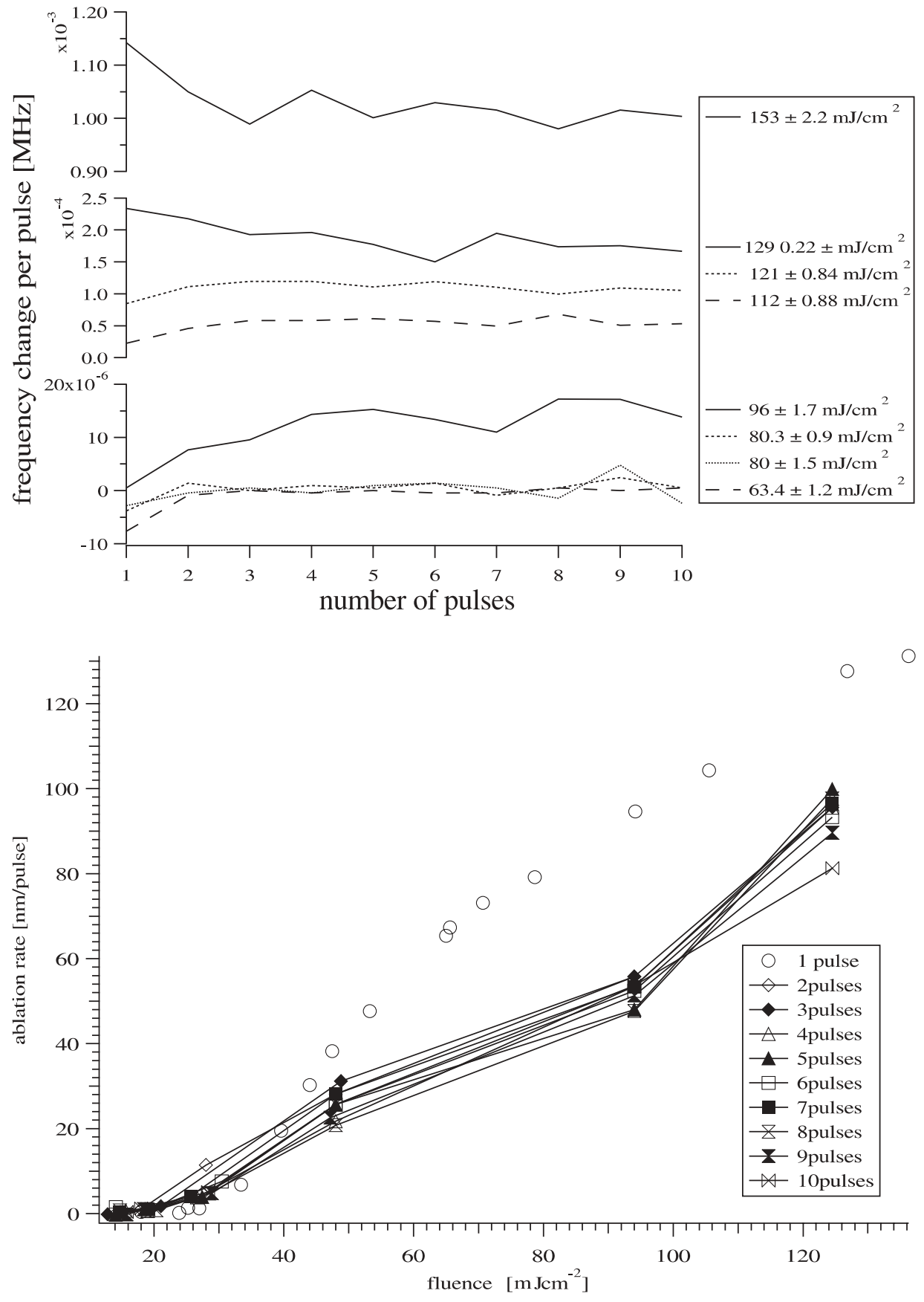

FIGURE 2 Frequency change between pulses at different fluences for PMDA at an irradiation wavelength of $308 \mathrm{~nm}$. One sample was used for each measurement at given fluence
FIGURE 3 Ablation behavior of TP polymer for $248 \mathrm{~nm}$ irradiation. Dependence of the pulse ablation rate on the pulse number
The behavior of PMDA after irradiation at a wavelength of $308 \mathrm{~nm}$ is quite different. A clear drop in the frequency change from the first to the later pulses is observed between 129 and $153 \mathrm{mJcm}^{-2}$ (Fig. 2). For irradiation with fluences between 96 and $121 \mathrm{mJcm}^{-2}$, however, an opposite trend (increase) of the frequency change is observed. This means that more material is removed with pulses following the first pulse. The reason for this behavior is not yet fully understood but could be related to the preparation of the sample, where a dense skin layer may be formed [9].

The corresponding ablation rates of TP for various fluences at an irradiation wavelength of $248 \mathrm{~nm}$, derived from the QCM measurements, are shown in Fig. 3. The curves show a significant difference of the ablation rate for the first pulse and the subsequent pulses, indicating that the ablation rate of the polymer at this wavelength will vary depending on the number of pulses applied at a given position. It is especially noteworthy that the ablation rates of the following pulses seem to approach the first pulse rates for higher fluences. A similar effect was found for the ablation behavior of PMDA at $308 \mathrm{~nm}$. Effective removal of the carbonized layer by high fluence pulses is a likely explanation.

These results show clearly that data obtained for the ablation rate of a polymer using just one quartz sample for irradiation with various fluences, and therefore a large number of pulses, are very probably the superposition of the ablation of the material, carbon and carbonized ma- 


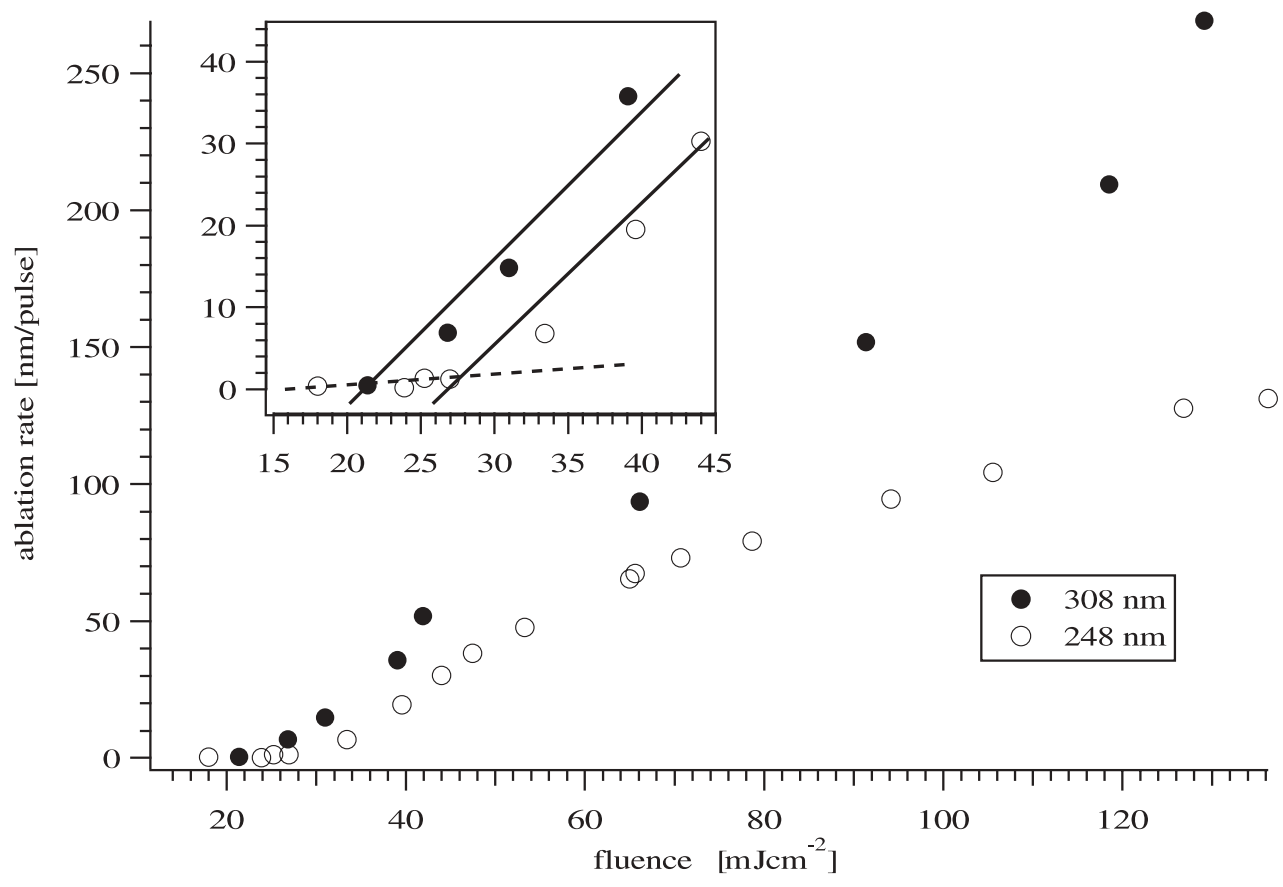

FIGURE 4 Ablation rate of TP for 248 and $308 \mathrm{~nm}$ irradiation. The ablation rate calculated from the QCM measurements with single pulse and a new sample for each experiment

terial. The experiments for TP at $308 \mathrm{~nm}$ did not show such a strong deviation for the subsequent pulses, which is probably due to the fact that carbonization is not very pronounced at this wavelength.

The ablation rates of TP at the two different wavelengths (248 and $308 \mathrm{~nm}$ ) are shown in Fig. 4. As expected, a clear dependence of the ablation rate on the laser wavelength with similar ablation thresholds are observed, confirming data observed for previous measurements with other techniques [10]. A clear Arrhenius tail (dashed line in the inset of Fig. 4) is observed for TP for an irradiation wavelength of $248 \mathrm{~nm}$ (open circles in Fig. 4) but is, however, not detected for an irradiation wavelength of $308 \mathrm{~nm}$ (filled circles). The Arrhenius tail refers to the change in the slope of the ablation curve marked by the lines. In the paper of Küper, a clear Arrhenius tail is observed for Kapton ${ }^{\mathrm{TM}}$ at all irradiation wavelengths except $193 \mathrm{~nm}$. This has been assigned to the highest photon energy, which may cause the photochemical decomposition. It is therefore noteworthy that TP shows the Arrhenius tail at shorter irradiation wavelengths with the higher photon energy and not at the longer irradiation wavelength with the lower photon energy. This can be attributed to the photodecomposition of the triazene groups in $\mathrm{TP}$, which have the highest absorption at an irradiation wavelength of $308 \mathrm{~nm}$.

The small changes at low fluences are most probably due to a mass loss without clear ablation. Two sources are probably responsible: evaporation of solvent and/or a linear photodecomposition of the triazene groups, which results in the loss of $\mathrm{N}_{2}$ (e.g., observed in solution with very low fluences) [11].

The results show clearly that the polymer surfaces are already modified with the first pulse and that the ablation rate changes significantly with successive pulses. It is not yet fully understood how this difference will affect the models that ap- ply data from single-pulse experiments but with consecutive pulses on the same position.

\section{$4 \quad$ Conclusion}

Measurements of the thickness changes (QCM frequency change) between successive pulses at the same fluence on the same sample show a strong deviation from the values obtained for the first pulse. This is due to the carbonization of the polymer surface, which takes place with the first pulse. This implies that the QCM data from previous studies do not give a very accurate number for ablation rates. This may influence the theoretical models that are based on such data. The QCM is a very valuable technique for studying the ablation behavior of various materials.

ACKNOWLEDGEMENTS Support of the Marie Curie Fellowship as well as the help of the laboratory members of the Laboratoire de Physico-Chimie Moléculaire, Université Bordeaux1 is gratefully acknowledged.

\section{REFERENCES}

1 D. Bäuerle: Laser Processing and Chemistry, 3rd edn (Springer Berlin Heidelberg 2000)

2 T. Lippert, J.T. Dickinson: Chem. Rev. 103, 453 (2003)

3 G.Z. Sauerbrey: Z. Phys. 155, 206 (1959)

4 S. Küper, J. Brannon, K. Brannon: Appl. Phys. A 56, 43 (1993)

5 T. Lippert, T. Nakamura, H. Niino, A. Ouchi, A. Yabe: Macromolecules 29, 6301 (1996)

6 F. Raimondi, S. Abolhassani, R. Brütsch, F. Geiger, T. Lippert, J. Wambach, J. Wei, A. Wokaun: J. Appl. Phys. 88, 3659 (2000)

7 N. Arnold, N. Bityurin: Appl. Phys. A 68, 615 (1999)

8 N. Bityurin, B. S. Luk'yanchuk, M.H. Hong, T.C. Chong: Chem. Rev. 103, 519 (2003)

9 T. Lippert, R.L. Webb, S.C. Langford, J.T. Dickinson: J. Appl. Phys. 85, 1838 (1999)

10 T. Lippert, L.S. Bennett, T. Nakamura, H. Niino, A. Ouchi, A. Yabe: Appl. Phys. A 63, 257 (1996)

11 J, Wei, N. Hoogen, T. Lippert, O. Nuyken, A. Wokaun: J. Phys. Chem. B 105, 1267 (2001) 